\title{
Nitroketene dithioacetal chemistry: Synthesis of coumarins incorporating nitrothiophene moiety
}

\author{
H SURYA PRAKASH RAO* and K VASANTHAM \\ Department of Chemistry, Pondicherry University, Pondicherry 605 014, India \\ e-mail: hspr.che@pondiuni.edu.in
}

MS received 7 September 2010; revised 14 December 2010; accepted 22 December 2010

\begin{abstract}
Alkylation of dipotassium 2-nitro-1,1-ethylenedithiolate 1 with ethyl 4-chloroacetoacetate 6 in a mixture of $\mathrm{MeOH}-\mathrm{H}_{2} \mathrm{O}$ (2:1) furnished highly functionalized 3-nitrothiophene 5. Pechmann condensation of 5 with 2-hydroxybenzaldehydes $\mathbf{8 a - i ,} 10$ furnished coumarin-3-nitrothiophene conjugates $\mathbf{9 a - i ,} 11$.
\end{abstract}

Keywords. Nitroketenedithioacetals; 3-nitrothiophenes; 2-hydroxy benzaldehydes; coumarins; Pechmann condensation.

\section{Introduction}

Nitroketene dithioacetals $\mathbf{2}$, prepared from carbon disulfide, nitromethane and alkylating agents in a two-step process, are extremely useful two carbon synthons for the synthesis of heterocyclic compounds incorporating diverse functional groups. ${ }^{1}$ We have been investigating on the alkylation of dipotassium salt of 2-nitro-1, 1-ethylenedithiolate $\mathbf{1}$ with a variety of alkyl halides and found that product formation is critically dependent on the nature of the alkyl halide and conditions. We found that while alkylation of the salt $\mathbf{1}$ with simple alkyl halides provided bis-alkylated products of the type 2 (route a, scheme 1 ), ${ }^{2}$ alkylation with sterically hindered alkyl halides or with propargyl bromide provided 1,3-dithioles of the type $\mathbf{3}$ (route $b$, scheme 1) and 4 (route c, scheme 1) respectively. ${ }^{3,4}$ Recently we found that alkylation of the salt 1 with acyl methyl chlorides furnished 3-nitrothiophenes of the type $\mathbf{5}$ (route d, scheme 1$){ }^{5}$

In continuation of above studies, we considered the reaction of the salt $\mathbf{1}$ with an alkyl halide having multiple functionalities, like ethyl 4-chloroacetoacetate $\mathbf{6}$, which is having two carbonyls, two active methylenes and two leaving groups namely chloro and ethoxy. The reaction could produce thiophene $\mathbf{5}$ going via the route $\mathrm{d}$ in analogy with our previous findings involving acyl methyl chlorides or it could produce mono- or bis-alkylated products (route a or b) followed by cyclization. To evaluate these interesting possibilities, we performed alkylation of $\mathbf{6}$ with the salt $\mathbf{1}$. The

*For correspondence reaction provided a single product, ethyl 4-\{[4-(2ethoxy-2-oxoethyl)-3-nitrothiophen-2-yl]sulfanyl \}-3oxobutanoate 5 ( $\mathrm{R}=\mathrm{COOEt}$; scheme 1$)$. The product was obviously formed via route $\mathrm{d}$ (scheme 1 ). In this paper, we give details of this study for the synthesis of highly functionalized 3-nitrothiophene 5. Thiophenes in general ${ }^{6}$ and 3-nitrothiophenes ${ }^{7}$ in particular have found several applications in pharmaceutical and technological fields. Further to isolation and characterization of 3-nitrothiophene 5, we describe its transformation into two more 3-nitrothiophene derivatives and nine coumarin 3-nitrothiophene conjugates.

\section{Experimental}

\subsection{General}

Reactions were performed in oven-dried glassware $\left(150^{\circ} \mathrm{C}\right)$. Dichloromethane, tetrahydrofuran, hexanes, ethyl acetate obtained commercially were distilled before use. Thin layer chromatography (TLC) was carried out using silica gel $60 \mathrm{~F}_{254}$ plates. The developed chromatogram was analysed by UV lamp $(254 \mathrm{~nm})$ or iodine vapors. The crude compounds were purified by flash chromatography on silica gel (230400 mesh) using hexanes-EtOAc mixture (10\% to $50 \%$ EtOAc) as eluent. Ethyl 4-chloroacetoacetate, 2-hydroxybenzaldehyde (salicylaldehyde) and piperidine were purchased from Sigma Aldrich and used as received. Substituted 2-hydroxybenzaldehydes $\mathbf{8 b} \mathbf{b}-\mathbf{i}$ were prepared following the procedure described by Wynberg. ${ }^{8}$ The ${ }^{1} \mathrm{H}$ NMR (400 MHz/300 MHz/60 MHz) 


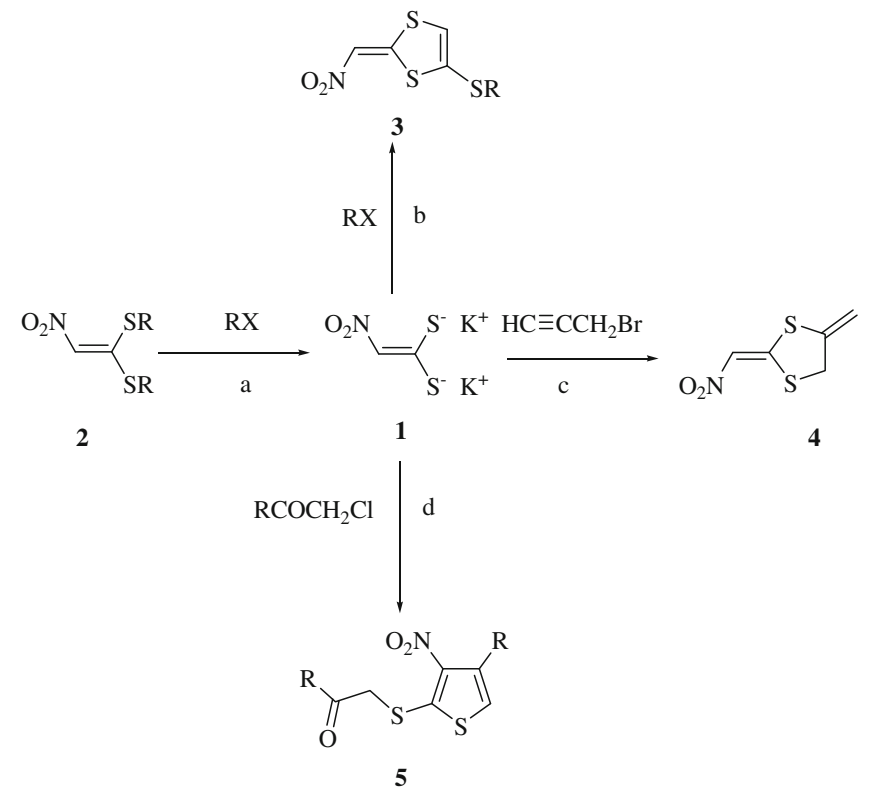

Scheme 1. Reaction of dipotassium salt $\mathbf{1}$ with different alkyl halides.

and $\left.{ }^{13} \mathrm{C} \mathrm{NMR} \mathrm{(100} \mathrm{MHz} \mathrm{/} 75 \mathrm{MHz}\right)$, DEPT spectra were recorded in $\mathrm{CDCl}_{3} / \mathrm{CDCl}_{3}: \mathrm{CCl}_{4}$ (1:1)/DMSO- $d_{6}: \mathrm{CCl}_{4}$ (1:1) on Bruker $400 \mathrm{MHz}$, JEOL $300 \mathrm{MHz}$ or JEOL $60 \mathrm{MHz}$ FT-NMR spectrometers with tetramethylsilane (TMS; 0 ppm) as internal standard. CHN analysis were performed on PerkinElmer elemental analyzer.

2.2 Preparation of ethyl 4-\{[4-(2-ethoxy-2-oxoethyl)3-nitro-2-thienyl]sulfanyl\}-3-oxobutanoate 5a<smiles>CCOC(=O)CSc1scc(CC(=O)CC(=O)OCC)c1[N+](=O)[O-]</smiles>

$5 a$

To a stirred suspension of freshly prepared dipotassium salt of nitroketene dithioacetate $1(0.5 \mathrm{~g}, 2.35 \mathrm{mmol})$ in a mixtureof $\mathrm{MeOH}$ and water $(2: 1 ; 10 \mathrm{~mL})$ at $0^{\circ} \mathrm{C}$ a dilute solution of ethyl 4-chloro-3-oxobutanoate $6(0.77 \mathrm{~g}, 4.70 \mathrm{mmol})$ in aqueous $\mathrm{MeOH}(15 \mathrm{~mL})$ was added by using pressure equalizer funnel at $0^{\circ} \mathrm{C}$ during $45 \mathrm{~min}$. The resulting reaction mixture was then stirred vigorously at room temperature (rt) for $6 \mathrm{~h}$. After the completion of the reaction (TLC; hexanes - EtOAc $=6: 4$ ), the mixture was transferred into a beaker containing $20 \mathrm{~g}$ of crushed ice. The acidic $(\mathrm{pH}=5)$ reaction mixture was carefully neutralized with $0.1 \mathrm{~N} \mathrm{NaHCO}_{3}$. The contents of the reaction mixture separated into two phases on dilution with dichloromethane $(45 \mathrm{~mL})$. The organic layer was washed with water $(3 \times 25 \mathrm{~mL})$ and brine $(2 \times$ $15 \mathrm{~mL}$ ) and dried over anhydrous $\mathrm{Na}_{2} \mathrm{SO}_{4}$. Evaporation of the solvent under reduced pressure resulted in the crude product as dark brown pasty mass. The crude product was subjected to column chromatography on silica gel by using increasing amounts of EtOAc (5\% to $40 \%$ ) in hexanes as eluent. Evaporation of the pooled fraction having required 3-nitrothiophene furnished $1.14 \mathrm{~g}$ of ethyl 4-\{[4-(2-ethoxy-2-oxoethyl)-3nitro-2-thienyl]sulfanyl\}-3-oxobutanoate $\mathbf{5 a}$ as yellow crystalline solid in $57 \%$ yield. $\mathrm{Mp}: 79-80^{\circ} \mathrm{C}(\mathrm{MeOH})$; UV $\lambda_{\max }(\mathrm{MeOH}): 281 \mathrm{~nm}(\log \varepsilon=4.1), 370 \mathrm{~nm}(\log$ $\varepsilon=3.6)$; IR $v_{\max }(\mathrm{KBr}): 1722(\mathrm{CO}), 1547,1489,1370$ $\left(\mathrm{NO}_{2}\right), 1081,768 \mathrm{~cm}^{-1} .{ }^{1} \mathrm{H}$ NMR $\delta\left(400 \mathrm{MHz} ; \mathrm{CDCl}_{3}\right.$; $\left.\mathrm{Me}_{4} \mathrm{Si}\right): 7.01(\mathrm{~s}, 1 \mathrm{H}, \mathrm{CH}), 4.23-4.14\left(\mathrm{~m}, 4 \mathrm{H}, \mathrm{OCH}_{2}\right)$, $4.11\left(\mathrm{~s}, 2 \mathrm{H}, \mathrm{CH}_{2}\right), 3.85\left(\mathrm{~s}, 2 \mathrm{H}, \mathrm{CH}_{2}\right), 3.66\left(\mathrm{~s}, 2 \mathrm{H}, \mathrm{CH}_{2}\right)$, 1.29 (t, $\left.J=7.2 \mathrm{~Hz}, 3 \mathrm{H}, \mathrm{CH}_{3}\right), 1.27$ (t, $J=7.2 \mathrm{~Hz}, 3 \mathrm{H}$, $\left.\mathrm{CH}_{3}\right) ;{ }^{13} \mathrm{C}$ NMR $\delta\left(100 \mathrm{MHz} ; \mathrm{CDCl}_{3} ; \mathrm{Me}_{4} \mathrm{Si}\right): 195.7$ (C), 169.7 (C), 166.4 (C), 147.5 (C), 141.8 (C), 131.5 (C), $121.5(\mathrm{CH}), 61.7\left(\mathrm{CH}_{2}\right), 61.1\left(\mathrm{CH}_{2}\right), 47.3\left(\mathrm{CH}_{2}\right)$, $44.9\left(\mathrm{CH}_{2}\right), 36.2\left(\mathrm{CH}_{2}\right), 14.2\left(\mathrm{CH}_{3}\right), 14.1\left(\mathrm{CH}_{3}\right) ; \mathrm{HRMS}$ $\left(\mathrm{ESI}^{+}\right)$: calcd for $\mathrm{C}_{14} \mathrm{H}_{17} \mathrm{NNaO}_{7} \mathrm{~S}_{2}\left(\mathrm{MNa}^{+}\right)$, 398.0344; found, 398.0344. Anal. Calcd. for $\mathrm{C}_{14} \mathrm{H}_{17} \mathrm{NO}_{7} \mathrm{~S}_{2}$ : C 44.71; H, 4.56; N, 3.71; S, 17.08; found: C 44.68; H, $4.52 ; \mathrm{N}, 3.69 ; \mathrm{S}, 17.06$.

2.2a X-ray crystal structure of ethyl 4-[4-(2-ethoxy2-oxoethyl)-3-nitro-2-thienyl]sulfanyl-3-oxobutanoate 5a:

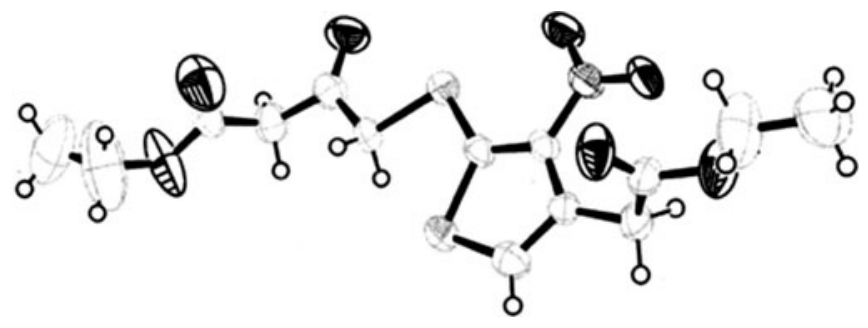

Crystal data and structure refinement data for $\mathbf{5 a}$.

Identification code

Empirical formula

Formula weight

Temperature

: CCDC 633059

Wavelength

Crystal system, space group
: ' $\mathrm{C}_{14} \mathrm{H}_{17} \mathrm{NO}_{7} \mathrm{~S}_{2}$ '

$: 375.41$

$: 273(2)$

: 0.71073 Á

: Monoclinic P2 1 
Unit cell

dimensions

Volume

Z, Calculated density

Absorption coefficient

$\mathrm{F}(000)$

Crystal size

Theta range

for data

collection

Limiting indices

Reflections
collected /
unique

Completeness to theta $=25.00$

Absorption correction

Max. and min. transmission method

Data / restraints / parameters

Goodness-of-fit on $\mathrm{F}^{\wedge} 2$

Final $\mathrm{R}$ indices [I $>2 \operatorname{sigma}(\mathrm{I})]$

$\mathrm{R}$ indices (all data)

Largest diff. peak and hole
Refinement

$$
\begin{aligned}
& \text { : a = 14.9609(16) А́; } \\
& \alpha=90 \text { deg. } \\
& : \mathrm{b}=4.9763(5) \text { Á; } \\
& \beta=92.706 \text { (4) deg. } \\
& : \mathrm{c}=23.301(3) \AA \dot{A} \text {; } \\
& \gamma=90.00 \mathrm{deg} \text {. }
\end{aligned}
$$$$
\text { : 1732.8(3) } \AA^{3}
$$$$
\text { : } 4,1.525 \mathrm{Mg} / \mathrm{m}^{3}
$$

: $0.342 \mathrm{~mm}^{-1}$

: 784

$: 0.26 \times 0.11 \times 0.08 \mathrm{~mm}$

: 2.63 to $18.97 \mathrm{deg}$.

$$
\begin{aligned}
& :-16<=\mathrm{h}<=20 \\
& -4<=\mathrm{k}<=6 \\
& -33<=l<=31 \\
& : 16260 / 2949 \\
& {[\mathrm{R} \text { (int) }=0.0338]} \\
& : 99.9 \%
\end{aligned}
$$

: Semi-empirical from equivalents

: 0.9112 and 0.8730

$$
\begin{aligned}
& : \text { Full-matrix } \\
& \text { least-squares on } \mathrm{F}^{2} \\
& : 6821 / 1 / 437 \\
& : 0.971
\end{aligned}
$$

$: \mathrm{R} 1=0.0715$,

$$
\mathrm{wR} 2=0.1752
$$$$
: \mathrm{R} 1=0.0765 \text {, }
$$$$
\mathrm{wR} 2=0.1772
$$

$: 1.152$ and

-0.425 e. $\AA^{-3}$

2.2b Ethyl 2-\{4-nitro-5-[(2-oxopropyl)sulfanyl]-3thienyl\}acetate 7 :<smiles>CCOC(=O)Cc1csc(SCC(C)=O)c1[N+](=O)[O-]</smiles>

To a stirred solution of ethyl 4-\{[4-(2-ethoxy-2oxoethyl)-3-nitro-2-thienyl]sulfanyl $\}$-3-oxobutanoate 5a $(0.05 \mathrm{~g}, 0.13 \mathrm{mmol})$ in EtOH $(1 \mathrm{~mL}), 1$ drop of dil. $\mathrm{H}_{2} \mathrm{SO}_{4}$ (1 drop of conc. $\mathrm{H}_{2} \mathrm{SO}_{4}$ in $1 \mathrm{~mL} \mathrm{EtOH}$ ) was added. The resulting reaction mixture was refluxed on a pre-heated oil bath maintained at $100^{\circ} \mathrm{C}$ for $18 \mathrm{~h}$ for complete transformation (TLC hexanes - EtOAc 7:3). Excess acid was quenched with $\mathrm{Na}_{2} \mathrm{CO}_{3}$. Evaporation of the solvent under reduced pressure furnished $0.04 \mathrm{~g}$ of ethyl 2-\{4-nitro-5-[(2-oxopropyl)sulfanyl]3-thienyl bacetate 7 in $99 \%$ yield. $\mathrm{Mp}: 134-136^{\circ} \mathrm{C}$ $(\mathrm{MeOH}) ; \mathrm{UV} \lambda_{\max }(\mathrm{MeOH}): 238 \mathrm{~nm}(\log \varepsilon=4.1)$, $377 \mathrm{~nm}(\log \varepsilon=3.6)$; IR $\gamma_{\max }(\mathrm{KBr}): 1735(\mathrm{CO}), 1718$ (CO), 1546, 1492, $1319\left(\mathrm{NO}_{2}\right), 1093,863,738 \mathrm{~cm}^{-1}$. ${ }^{1} \mathrm{H}$ NMR $\delta\left(400 \mathrm{MHz} ; \mathrm{CDCl}_{3} ; \mathrm{Me}_{4} \mathrm{Si}\right): 6.99$ (s, $1 \mathrm{H}$, $\mathrm{CH}), 4.17\left(\mathrm{q}, J=7.2 \mathrm{~Hz}, 2 \mathrm{H}, \mathrm{CH}_{2}\right), 3.92(\mathrm{~s}, 1 \mathrm{H}, \mathrm{OH})$, $3.87\left(\mathrm{~s}, 3 \mathrm{H}, \mathrm{CH}_{3}\right), 2.37\left(\mathrm{~s}, 3 \mathrm{H}, \mathrm{CH}_{3}\right), 1.27(\mathrm{t}, J=$ $\left.7.2 \mathrm{~Hz}, 3 \mathrm{H}, \mathrm{CH}_{3}\right) ;{ }^{13} \mathrm{C}$ NMR $\delta\left(100 \mathrm{MHz} ; \mathrm{CDCl}_{3}\right.$; $\mathrm{Me}_{4} \mathrm{Si}$ ): 200.6 (C), 169.8 (C), 154.0 (C), 148.2 (C), $131.6(\mathrm{C}), 121.3(\mathrm{CH}), 61.2\left(\mathrm{CH}_{2}\right), 45.6\left(\mathrm{CH}_{2}\right), 36.3$ $\left(\mathrm{CH}_{2}\right), 28.6\left(\mathrm{CH}_{3}\right) 14.2\left(\mathrm{CH}_{3}\right)$. HRMS $\left(\mathrm{ESI}^{+}\right)$: calcd for $\mathrm{C}_{11} \mathrm{H}_{13} \mathrm{NNaO}_{5} \mathrm{~S}_{2}\left(\mathrm{MNa}^{+}\right)$, 326.0133; found, 326.0132. Anal. Calcd. for $\mathrm{C}_{11} \mathrm{H}_{13} \mathrm{NO}_{5} \mathrm{~S}_{2}$ : C, 43.50; H, 4.32; N, 4.62; S, 21.14; found: C 43.49; H, 4.30; N, 4.60; S, 21.12 .

2.2c Methyl 2-\{4-nitro-5-[(2-oxopropyl)sulfanyl]-3thienyl\}acetate 7a:<smiles>CC(=O)CSc1scc(CC(C)=O)c1[N+](=O)[O-]</smiles>

$7 \mathbf{a}$

Following the above procedure ethyl 4\{[4-(2-ethoxy-2-oxoethyl)-3-nitro-2-thienyl]sulfanyl $\}$ 3 -oxobutanoate 5a $(0.13 \mathrm{mmol})$ in $\mathrm{MeOH}(1 \mathrm{~mL})$, was transformed into $0.036 \mathrm{~g}$ of methyl 2-\{4-nitro-5[(2-oxopropyl)sulfanyl]-3-thienyl $\}$ acetate $\mathbf{7 a}$ in $96 \%$ yield with dil. $\mathrm{H}_{2} \mathrm{SO}_{4}$. Mp: $108-110^{\circ} \mathrm{C}(\mathrm{MeOH}) ; \mathrm{UV}$ $\lambda_{\max }(\mathrm{MeOH}): 238 \mathrm{~nm}(\log \varepsilon=4.1), 377 \mathrm{~nm}(\log \varepsilon=$ 3.6); IR $v_{\max }(\mathrm{KBr}): 1735$ (COOEt), 1718 (CO), 1546, 1492, $1319\left(\mathrm{NO}_{2}\right), 1093,863,738 \mathrm{~cm}^{-1} \cdot{ }^{1} \mathrm{H}$ NMR $\delta$ $\left(400 \mathrm{MHz} ; \mathrm{CDCl}_{3} ; \mathrm{Me}_{4} \mathrm{Si}\right): 6.98(\mathrm{~s}, 1 \mathrm{H}, \mathrm{CH}), 3.89$ (s, $\left.2 \mathrm{H}, \mathrm{CH}_{2}\right), 3.87\left(\mathrm{~s}, 2 \mathrm{H}, \mathrm{CH}_{2}\right), 3.72\left(\mathrm{~s}, 3 \mathrm{H}, \mathrm{CH}_{3}\right)$, $2.37\left(\mathrm{~s}, 3 \mathrm{H}, \mathrm{CH}_{3}\right) ;{ }^{13} \mathrm{C}$ NMR $\delta\left(100 \mathrm{MHz} ; \mathrm{CDCl}_{3}\right.$; $\left.\mathrm{Me}_{4} \mathrm{Si}\right): 200.2(\mathrm{C}), 170.0(\mathrm{C} \times 2), 147.7(\mathrm{C}), 131.4$ (C), $121.2(\mathrm{CH}), 52.2\left(\mathrm{OCH}_{3}\right), 45.5\left(\mathrm{CH}_{2}\right), 36.0\left(\mathrm{CH}_{2}\right)$, $28.5\left(\mathrm{CH}_{3}\right)$. HRMS $\left(\mathrm{ESI}^{+}\right)$: calcd for $\mathrm{C}_{10} \mathrm{H}_{11} \mathrm{NNaO}_{5} \mathrm{~S}_{2}$ $\left(\mathrm{MNa}^{+}\right), 311.9976$; found, 311.9976. Anal. Calcd. for 
$\mathrm{C}_{10} \mathrm{H}_{11} \mathrm{NO}_{5} \mathrm{~S}_{2}$ : C, 41.51; H, 3.83; N, 4.84; S, 22.17; found: C 41.49; H, 3.80; N, 4.82; S, 22.12.

\subsection{Representative procedure for the synthesis} of coumarins: Preparation of ethyl 2-\{4-nitro-5-[(2oxo-2H-3-chromenyl)sulfanyl]-3-thienyl\}acetate $\mathbf{9 a}$

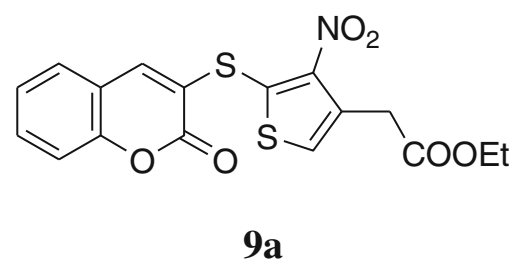

To a homogenous solution of 2-hydroxybenzaldehyde 8a $(0.02 \mathrm{~g}, 0.16 \mathrm{mmol})$ in THF, piperidine $(0.001 \mathrm{~g}$, $0.1 \mathrm{~mol} \%$ ) was added and stirred for $10 \mathrm{~min}$ at rt. The reaction mixture became brown in colour. To this solution, ethyl 4-\{[4-(2-ethoxy-2-oxoethyl)-3nitro-2-thienyl]sulfanyl $\}$-3-oxobutanoate 5a $(0.05 \mathrm{~g}$, $0.13 \mathrm{mmol}$ ) was added and the reaction mixture was allowed to stir at $\mathrm{rt}$ for $32 \mathrm{~h}$ for completion of the reaction (TLC: hexanes- EtOAc 8:2). The reaction mixture was then diluted with dichloromethane $(25 \mathrm{~mL})$ and the organic layer was washed sequentially with water $(3 \times 25 \mathrm{~mL})$, brine and dried over anhydrous sodium sulfate. Evaporation of the solvent under reduced pressure resulted in crude ethyl 2-\{4-nitro5-[(2-oxo-2H-3-chromenyl)sulfanyl]-3-thienyl $\}$ acetate 9a as gummy liquid. The crude product was subjected to column chromatography on $\mathrm{SiO}_{2}(35 \mathrm{~g}, 15 \mathrm{~cm} \times$ $1 \mathrm{~cm}$ ) using increasing amounts of ethyl acetate in hexanes as eluent. Evaporation of the pooled fractions having the required product resulted in $0.042 \mathrm{~g}$ of $\mathbf{9 a}$ as a light yellow colour solid in 69\% yield. M.p.: 188 $190^{\circ} \mathrm{C}(\mathrm{MeOH}) ; \mathrm{R}_{f}: 0.68$ (hexanes - EtOAc; 8:2); UV $\lambda_{\max }(\mathrm{MeOH}): 278 \mathrm{~nm}(\log \varepsilon=4.2), 374 \mathrm{~nm}(\log \varepsilon=$ 3.4); IR $\gamma_{\max }(\mathrm{KBr}): 1735(\mathrm{CO}), 1541,1492,1319$ $\left(\mathrm{NO}_{2}\right), 1096,881,729 \mathrm{~cm}^{-1}$. ${ }^{1} \mathrm{H}$ NMR $(300 \mathrm{MHz}$, $\left.\mathrm{CDCl}_{3} ; \mathrm{Me}_{4} \mathrm{Si}\right): \delta 8.22(\mathrm{~s}, 1 \mathrm{H}, \mathrm{CH}), 7.68-7.35(\mathrm{~m}$, $4 \mathrm{H}, \mathrm{CH}), 7.02(\mathrm{~s}, 1 \mathrm{H}, \mathrm{CH}), 4.18(\mathrm{q}, J=7.2 \mathrm{~Hz}, 2 \mathrm{H}$, $\left.\mathrm{OCH}_{2}\right), 3.89\left(\mathrm{~s}, 2 \mathrm{H}, \mathrm{CH}_{2}\right), 1.27(\mathrm{t}, J=7.2 \mathrm{~Hz}, 3 \mathrm{H}$, $\left.\mathrm{CH}_{3}\right) ;{ }^{13} \mathrm{C}$ NMR $\left(300 \mathrm{MHz}, \mathrm{CDCl}_{3} ; \mathrm{Me}_{4} \mathrm{Si}\right): \delta 169.8$ (C), $158.2(\mathrm{C}), 154.5(\mathrm{C}), 150.5(\mathrm{CH}), 148.7(\mathrm{C})$, $142.2(\mathrm{C}), 133.6(\mathrm{CH}), 131.2(\mathrm{C}), 128.5(\mathrm{CH}), 125.1$ $(\mathrm{CH}), 123.1(\mathrm{CH}), 120.8(\mathrm{C}), 118.7(\mathrm{C}), 117.0(\mathrm{CH})$, $61.3\left(\mathrm{CH}_{2}\right), 36.2\left(\mathrm{CH}_{2}\right), 14.1\left(\mathrm{CH}_{3}\right)$. HRMS $\left(\mathrm{ESI}^{+}\right)$: calcd for $\mathrm{C}_{17} \mathrm{H}_{13} \mathrm{NNaO}_{6} \mathrm{~S}_{2}\left(\mathrm{MNa}^{+}\right)$, 414.0082; found, 414.0079. Anal. calcd. for $\mathrm{C}_{17} \mathrm{H}_{13} \mathrm{NO}_{6} \mathrm{~S}_{2}: \mathrm{C}, 52.16 ; \mathrm{H}$, $3.35 ; \mathrm{N}, 3.58 ; \mathrm{S}, 16.38$; found: C 52.19; H, 3.31; N, 3.59; S, 16.36 . 2.3a Ethyl 2-\{5-[(6-chloro-2-oxo-2H-3chromenyl) sulfanyl]-4-nitro-3-thienyl\}acetate $\mathbf{9 b}$ :

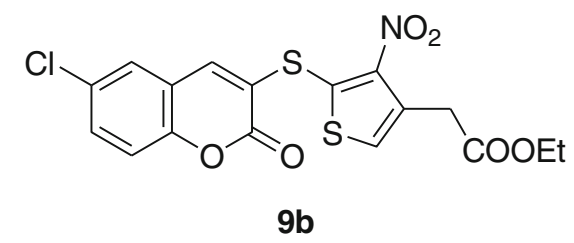

Following the general procedure described above, the reaction of 5-chloro-2-hydroxybenzaldehyde $\mathbf{8 b}$ $(0.024 \mathrm{~g}, 0.16 \mathrm{mmol})$ and ethyl 4-\{[4-(2-ethoxy2-oxoethyl)-3-nitro-2-thienyl]sulfanyl\}-3-oxobutanoate $5 \mathrm{a}(0.05 \mathrm{~g}, 0.13 \mathrm{mmol})$ in the presence of piperidine $(0.001 \mathrm{~g}, 0.1 \mathrm{~mol} \%)$ stirred at $\mathrm{rt}$ for $34 \mathrm{~h}$ furnished $0.037 \mathrm{~g}$ of ethyl 2-\{5-[(6-chloro-2-oxo- $2 \mathrm{H}$ 3-chromenyl)sulfanyl]-4-nitro-3-thienyl \}acetate $\quad 9 b$ as yellow colour solid in $66 \%$ yield. Mp: $160-161^{\circ} \mathrm{C}$ $(\mathrm{MeOH}) ; \mathrm{UV} \lambda_{\max }(\mathrm{MeOH}): 271 \mathrm{~nm}(\log \varepsilon=4.3)$, $379 \mathrm{~nm}(\log \varepsilon=3.8) ;$ IR $\gamma_{\max }(\mathrm{KBr}): 1740(\mathrm{CO}), 1546$, 1492, $1319\left(\mathrm{NO}_{2}\right), 1091,883,740 \mathrm{~cm}^{-1} .{ }^{1} \mathrm{H}$ NMR $\delta$ (300 MHz, $\mathrm{CDCl}_{3}$ : DMSO- $\left.d_{6} ; \mathrm{Me}_{4} \mathrm{Si}\right): 8.35(\mathrm{~s}, 1 \mathrm{H}$, $\mathrm{CH}), 7.72(\mathrm{~s}, 1 \mathrm{H}, \mathrm{CH}), 7.63(\mathrm{~d}, J=8.7 \mathrm{~Hz}, 1 \mathrm{H}, \mathrm{CH})$, $7.39(\mathrm{~d}, J=9 \mathrm{~Hz}, 1 \mathrm{H}, \mathrm{CH}), 7.27(\mathrm{~s}, 1 \mathrm{H}, \mathrm{CH}), 4.15$ (q, $\left.J=7.2 \mathrm{~Hz}, 2 \mathrm{H}, \mathrm{OCH}_{2}\right), 3.90\left(\mathrm{~s}, 2 \mathrm{H}, \mathrm{CH}_{2}\right), 1.26(\mathrm{t}$, $\left.J=7.2 \mathrm{~Hz}, 3 \mathrm{H}, \mathrm{CH}_{3}\right) ;{ }^{13} \mathrm{C} \mathrm{NMR} \delta\left(300 \mathrm{MHz}, \mathrm{CDCl}_{3}\right.$ : DMSO- $d_{6} ; \mathrm{Me}_{4} \mathrm{Si}$ ): 169.3 (C), 157.4 (C), 152.2 (C), $148.6(\mathrm{CH}), 143.7(\mathrm{C}), 142.5(\mathrm{C}), 132.9(\mathrm{CH}), 130.7$ (C), $129.7(\mathrm{C}), 127.6(\mathrm{CH}), 124.2(\mathrm{CH}), 121.7(\mathrm{C})$, $119.5(\mathrm{C}), 117.9(\mathrm{CH}), 60.6\left(\mathrm{CH}_{2}\right), 35.6\left(\mathrm{CH}_{3}\right), 13.8$ $\left(\mathrm{CH}_{3}\right)$. HRMS $\left(\mathrm{ESI}^{+}\right)$: calcd for $\mathrm{C}_{17} \mathrm{H}_{12} \mathrm{ClNNaO}_{6} \mathrm{~S}_{2}$ $\left(\mathrm{MNa}^{+}\right)$, 447.9692; found, 447.9677. Anal. Calcd. for $\mathrm{C}_{17} \mathrm{H}_{12} \mathrm{ClNO}_{6} \mathrm{~S}_{2}: \mathrm{C}, 47.95 ; \mathrm{H}, 2.84 ; \mathrm{Cl}, 8.32 ; \mathrm{N}, 3.29$; S, 15.06; found: C, 47.91; H, 2.79; Cl, 8.30; N, 3.31; S, 15.09 .

\section{3b Ethyl 2-\{5-[(6-bromo-2-oxo-2H-3-} chromenyl) sulfanyl]-4-nitro-3-thienyl\}acetate 9c:

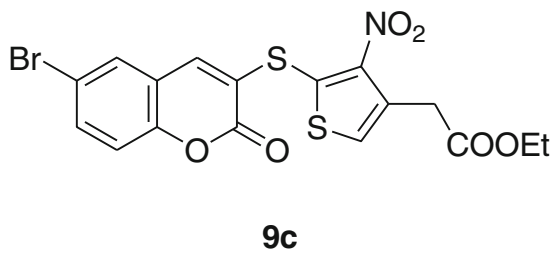

Following the general procedure described above, the reaction of 5-bromo-2-hydroxybenzaldehyde $\mathbf{8 c}$ $(0.032 \mathrm{~g}, 0.16 \mathrm{mmol})$ and ethyl 4-\{[4-(2-ethoxy2-oxoethyl)-3-nitro-2-thienyl]sulfanyl\}-3-oxobutanoate $5 \mathbf{a}(0.05 \mathrm{~g}, 0.13 \mathrm{mmol})$ in the presence of piperidine $(0.001 \mathrm{~g}, 0.1 \mathrm{~mol} \%)$ stirred at $\mathrm{rt}$ for $34 \mathrm{~h}$ furnished $0.028 \mathrm{~g}$ of ethyl 2-\{5-[(6-bromo-2-oxo-2H-3-chromenyl) 
sulfanyl]-4-nitro-3-thienyl \}acetate 9c as yellow colour solid in $48 \%$ yield. $\mathrm{Mp}: 170-172^{\circ} \mathrm{C}(\mathrm{MeOH})$; UV $\lambda_{\max }(\mathrm{MeOH}): 276 \mathrm{~nm}(\log \varepsilon=4.3), 374 \mathrm{~nm}(\log \varepsilon=$ 3.8); IR $v_{\max }(\mathrm{KBr}): 1740(\mathrm{CO}), 1546,1492,1319$ $\left(\mathrm{NO}_{2}\right), 1091,883,740 \mathrm{~cm}^{-1} \cdot{ }^{1} \mathrm{H}$ NMR $\delta(300 \mathrm{MHz}$, $\mathrm{CDCl}_{3}:$ DMSO- $\left.d_{6} ; \mathrm{Me}_{4} \mathrm{Si}\right): 8.2(\mathrm{~s}, 1 \mathrm{H}, \mathrm{CH}), 7.73(\mathrm{~d}$, $J=9.0 \mathrm{~Hz}, 1 \mathrm{H}, \mathrm{CH}), 7.53(\mathrm{~s}, 1 \mathrm{H}, \mathrm{CH}), 7.32(\mathrm{~d}, J=$ $8.4 \mathrm{~Hz}, 1 \mathrm{H}, \mathrm{CH}), 7.19(\mathrm{~s}, 1 \mathrm{H}, \mathrm{CH}), 4.17(\mathrm{q}, J=7.2 \mathrm{~Hz}$, $\left.2 \mathrm{H}, \mathrm{CH}_{2}\right), 3.91\left(\mathrm{~s}, 2 \mathrm{H}, \mathrm{CH}_{2}\right), 1.27(\mathrm{t}, J=7.2 \mathrm{~Hz}, 3 \mathrm{H}$, $\left.\mathrm{CH}_{3}\right) ;{ }^{13} \mathrm{C}$ NMR $\delta\left(300 \mathrm{MHz}, \mathrm{CDCl}_{3} ; \mathrm{Me}_{4} \mathrm{Si}\right): 169.9$ (C), $157.6(\mathrm{C}), 153.0(\mathrm{C}), 150.9(\mathrm{CH}), 146.4(\mathrm{C}), 141.3$ (C), $136.2(\mathrm{CH}), 131.6(\mathrm{CH}), 130.7(\mathrm{C}), 128.6(\mathrm{CH})$, $125.0(\mathrm{CH}), 120.4(\mathrm{C}), 120.3(\mathrm{C}), 118.7(\mathrm{CH}), 60.5$ $\left(\mathrm{OCH}_{2}\right), 35.4\left(\mathrm{CH}_{2}\right), 13.8\left(\mathrm{CH}_{3}\right)$. HRMS $\left(\mathrm{ESI}^{+}\right)$: calcd for $\mathrm{C}_{17} \mathrm{H}_{12} \mathrm{BrNNaO}_{6} \mathrm{~S}_{2}\left(\mathrm{MNa}^{+}\right), 491.9187$; found, 491.9172. Anal. Calcd. for $\mathrm{C}_{17} \mathrm{H}_{12} \mathrm{BrNO}_{6} \mathrm{~S}_{2}$ : C, 43.41; H, 2.57; Br, 16.99; N, 2.98; S, 13.64; found: C, 43.40; H, 2.59; Br, 17.01; N, 2.99; S, 13.68 .

2.3c Ethyl 2-\{5-[(6-methoxy-2-oxo-2H-3chromenyl) sulfanyl]-4-nitro-3-thienyl)acetate 9d:<smiles>CCOC(=O)Cc1csc(Sc2cc3cc(OC)ccc3oc2=O)c1[N+](=O)[O-]</smiles>

9d

Following the general procedure described above, the reaction of 5-methoxy-2-hydroxybenzaldehyde 8d $(0.025 \mathrm{~g}, 0.17 \mathrm{mmol})$ and ethyl 4-\{[4-(2-ethoxy2-oxoethyl)-3-nitro-2-thienyl]sulfanyl \}-3-oxobutanoate $5 \mathbf{a}(0.052 \mathrm{~g}, 0.14 \mathrm{mmol})$ in the presence of piperidine $(0.001 \mathrm{~g}, 0.1 \mathrm{~mol} \%)$ stirred at $\mathrm{rt}$ for $32 \mathrm{~h}$ furnished $0.032 \mathrm{~g}$ of Ethyl 2-\{5-[(6-methoxy-2-oxo-2H-3chromenyl)sulfanyl]-4-nitro-3-thienyl \}acetate $\mathbf{9 d}$ as yellow colour solid in $53 \%$ yield. Mp: $170-172^{\circ} \mathrm{C}$ $(\mathrm{MeOH}) ; \mathrm{UV} \lambda_{\max }(\mathrm{MeOH}): 273 \mathrm{~nm}(\log \varepsilon=4.3)$, $374 \mathrm{~nm}(\log \varepsilon=3.8)$; IR $\gamma_{\max }(\mathrm{KBr}): 1736(\mathrm{CO})$, 1546, 1492, 1319, 1091, 883, $740 \mathrm{~cm}^{-1} .{ }^{1} \mathrm{H}$ NMR $\delta$ (400 MHz, $\mathrm{CDCl}_{3} ; \mathrm{Me}_{4} \mathrm{Si}$ ): 8.17 (s, 1H, CH), 7.27 (s, $1 \mathrm{H}, \mathrm{CH}), 7.18(\mathrm{~d}, J=8.0 \mathrm{~Hz}, 1 \mathrm{H}, \mathrm{CH}), 7.11(\mathrm{~d}, J=$ $7.8 \mathrm{~Hz}, 1 \mathrm{H}, \mathrm{CH}), 7.0$ (s, $1 \mathrm{H}, \mathrm{CH}), 4.17$ (q, $J=7.2 \mathrm{~Hz}$, $\left.2 \mathrm{H}, \mathrm{CH}_{2}\right), 4.0$ (s, $\left.3 \mathrm{H}, \mathrm{CH}_{3}\right), 3.88\left(\mathrm{~s}, 2 \mathrm{H}, \mathrm{CH}_{2}\right), 1.27$ $\left(\mathrm{t}, J=7.2 \mathrm{~Hz}, 3 \mathrm{H}, \mathrm{CH}_{3}\right) ;{ }^{13} \mathrm{C} \mathrm{NMR} \delta(300 \mathrm{MHz}$, $\left.\mathrm{CDCl}_{3} ; \mathrm{Me}_{4} \mathrm{Si}\right): 169.7(\mathrm{C}), 157.7(\mathrm{C}), 150.4(\mathrm{CH})$, 147.3 (C), 145.4 (C), 144.2 (C), 142.9 (C), 131.2 (C), $125.0(\mathrm{CH}), 123.0(\mathrm{CH}), 121.3(\mathrm{C}), 119.6(\mathrm{CH}), 119.3$ (C), $115.2(\mathrm{CH}), 61.2\left(\mathrm{CH}_{2}\right), 56.3\left(\mathrm{OCH}_{3}\right), 36.1\left(\mathrm{CH}_{2}\right)$, 14.1 $\left(\mathrm{CH}_{3}\right)$. HRMS $\left(\mathrm{ESI}^{+}\right)$: calcd for $\mathrm{C}_{18} \mathrm{H}_{15} \mathrm{NNaO}_{7} \mathrm{~S}_{2}$ $\left(\mathrm{MNa}^{+}\right), 444.0188$; found, 444.0179. Anal. Calcd. for
$\mathrm{C}_{18} \mathrm{H}_{15} \mathrm{NO}_{7} \mathrm{~S}_{2}$ : C, 51.30; H, 3.59; N, 3.32; S, 15.22; found: C, 50.29; H, 3.62; N, 3.35; S, 15.19 .

2.3d Ethyl 2-\{5-[(6-methyl-2-oxo-2H-3chromenyl) sulfanyl]-4-nitro-3-thienyl lacetate 9e:

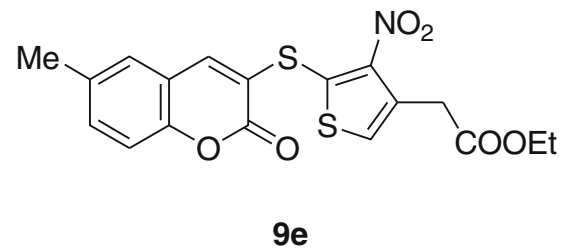

Following the general procedure described above, the reaction of 5-methyl-2-hydroxybenzaldehyde $\mathbf{8 e}$ $(0.044 \mathrm{~g}, 0.32 \mathrm{mmol})$ and ethyl 4-\{[4-(2-ethoxy2-oxoethyl)-3-nitro-2-thienyl]sulfanyl \}-3-oxobutanoate 5a $(0.1 \mathrm{~g}, 0.27 \mathrm{mmol})$ in the presence of piperidine $(0.001 \mathrm{~g}, 0.1 \mathrm{~mol} \%)$ stirred at $\mathrm{rt}$ for $36 \mathrm{~h}$ furnished $0.078 \mathrm{~g}$ of ethyl 2-\{5-[(6-methyl-2-oxo- $2 \mathrm{H}-$ 3-chromenyl)sulfanyl]-4-nitro-3-thienyl $\}$ acetate $9 e$ as yellow colour solid in $67 \%$ yield. Mp: $186-188^{\circ} \mathrm{C}$ $(\mathrm{MeOH}) ; \mathrm{UV} \lambda_{\max }(\mathrm{MeOH}): 276 \mathrm{~nm}(\log \varepsilon=4.3)$, $374 \mathrm{~nm}(\log \varepsilon=3.8)$; IR $\gamma_{\max }(\mathrm{KBr}): 1740(\mathrm{CO})$, 1546, 1492, 1319, 1091, 883, $740 \mathrm{~cm}^{-1}$. ${ }^{1} \mathrm{H}$ NMR $\delta$ $\left(400 \mathrm{MHz}, \mathrm{CDCl}_{3} ; \mathrm{Me}_{4} \mathrm{Si}\right): 8.2(\mathrm{~s}, 1 \mathrm{H}, \mathrm{CH}), 7.46(\mathrm{~d}$, $J=8.1 \mathrm{~Hz}, 1 \mathrm{H}, \mathrm{CH}), 7.33(\mathrm{~d}, J=8.7 \mathrm{~Hz}, 1 \mathrm{H}, \mathrm{CH})$, $7.28(\mathrm{~d}, J=6.6 \mathrm{~Hz}, 1 \mathrm{H}, \mathrm{CH}), 7.0(\mathrm{~s}, 1 \mathrm{H}, \mathrm{CH}), 4.15$ (q, $\left.J=7.2 \mathrm{~Hz}, 2 \mathrm{H}, \mathrm{OCH}_{2}\right), 3.97\left(\mathrm{~s}, 2 \mathrm{H}, \mathrm{CH}_{2}\right), 2.44$ (s, $\left.3 \mathrm{H}, \mathrm{CH}_{3}\right), 1.23\left(\mathrm{t}, J=7.2 \mathrm{~Hz}, 3 \mathrm{H}, \mathrm{CH}_{3}\right) ;{ }^{13} \mathrm{C} \mathrm{NMR} \delta$ $\left(100 \mathrm{MHz}, \mathrm{CDCl}_{3} ; \mathrm{Me}_{4} \mathrm{Si}\right): 169.9(\mathrm{C}), 158.1(\mathrm{C}), 152.3$ $(\mathrm{CH}), 148.2(\mathrm{C}), 140.7(\mathrm{C}), 135.0(\mathrm{CH}), 134.6(\mathrm{C} \times 2)$, $130.8(\mathrm{C}), 128.8(\mathrm{CH}), 124.6(\mathrm{CH}), 118.5(\mathrm{C}), 118.4$ (C), $116.2(\mathrm{CH}), 60.4\left(\mathrm{OCH}_{2}\right), 35.4\left(\mathrm{CH}_{2}\right), 20.1\left(\mathrm{CH}_{3}\right)$, 14.1 $\left(\mathrm{CH}_{3}\right)$. HRMS $\left(\mathrm{ESI}^{+}\right)$: calcd for $\mathrm{C}_{18} \mathrm{H}_{15} \mathrm{NNaO}_{6} \mathrm{~S}_{2}$ $\left(\mathrm{MNa}^{+}\right), 428.0238$; found, 428.0229. Anal. Calcd. for $\mathrm{C}_{18} \mathrm{H}_{15} \mathrm{NO}_{6} \mathrm{~S}_{2}$ : C, 53.32; H, 3.73; N, 3.43; $\mathrm{S}, 15.82$; found: C, 53.34; H, 3.71; N, 3.39; S, 15.79 .

\section{3e Ethyl 2-\{5-[(6-ethyl-2-oxo-2H-3-} chromenyl) sulfanyl]-4-nitro-3-thienyl\}acetate $9 \mathbf{f}$ :<smiles>CCOC(=O)Cc1csc(Sc2cc3cc(CC)ccc3oc2=O)c1[N+](=O)[O-]</smiles>

Following the general procedure described above, the reaction of 5-ethyl-2-hydroxybenzaldehyde $\mathbf{8 f}(0.024 \mathrm{~g}$, $0.16 \mathrm{mmol}$ ) and ethyl 4-\{[4-(2-ethoxy-2-oxoethyl)-3nitro-2-thienyl]sulfanyl\}-3-oxobutanoate 5a $(0.05 \mathrm{~g}$, 
$0.13 \mathrm{mmol})$ in the presence of piperidine $(0.001 \mathrm{~g}$, $0.1 \mathrm{~mol} \%$ ) stirred at $\mathrm{rt}$ for $36 \mathrm{~h}$ furnished $0.04 \mathrm{~g}$ of ethyl 2-\{5-[(6-ethyl-2-oxo-2H-3-chromenyl)sulfanyl]4-nitro-3-thienyl $\}$ acetate 9f as yellow colour solid in $69 \%$ yield. $\mathrm{Mp}: 174-176^{\circ} \mathrm{C}(\mathrm{MeOH})$; UV $\lambda_{\max }$ (MeOH): $274 \mathrm{~nm}(\log \varepsilon=4.1), 372 \mathrm{~nm}(\log \varepsilon=3.6)$; IR $v_{\max }(\mathrm{KBr}): 1738(\mathrm{CO}), 1546,1492,1319\left(\mathrm{NO}_{2}\right)$, $1091,883,740 \mathrm{~cm}^{-1} .{ }^{1} \mathrm{H}$ NMR $\delta\left(400 \mathrm{MHz}, \mathrm{CDCl}_{3}\right.$; $\left.\mathrm{Me}_{4} \mathrm{Si}\right): 8.17(\mathrm{~s}, 1 \mathrm{H}, \mathrm{CH}), 7.48(\mathrm{~d}, J=8.0 \mathrm{~Hz}, 1 \mathrm{H}, \mathrm{CH})$, $7.35(\mathrm{~d}, J=8.0 \mathrm{~Hz}, 1 \mathrm{H}, \mathrm{CH}), 7.32(\mathrm{~d}, J=8.0 \mathrm{~Hz}, 1 \mathrm{H}$, $\mathrm{CH}), 7.0(\mathrm{~s}, 1 \mathrm{H}, \mathrm{CH}), 4.18\left(\mathrm{q}, J=7.2 \mathrm{~Hz}, 2 \mathrm{H}, \mathrm{OCH}_{2}\right)$, $3.88\left(\mathrm{~s}, 2 \mathrm{H}, \mathrm{CH}_{2}\right), 2.73\left(\mathrm{q}, J=7.6 \mathrm{~Hz}, 2 \mathrm{H}, \mathrm{CH}_{2}\right), 1.29$ (t, $\left.J=6.0 \mathrm{~Hz}, 3 \mathrm{H}, \mathrm{CH}_{3}\right), 1.27\left(\mathrm{t}, J=5.2 \mathrm{~Hz}, 3 \mathrm{H}, \mathrm{CH}_{3}\right)$; ${ }^{13} \mathrm{C}$ NMR $\delta\left(100 \mathrm{MHz}, \mathrm{CDCl}_{3} ; \mathrm{Me}_{4} \mathrm{Si}\right): 169.7$ (C), $158.5(\mathrm{C}), 152.9(\mathrm{C} \times 2), 150.7(\mathrm{CH}), 141.3(\mathrm{C}), 133.7$ $(\mathrm{CH}), 131.2(\mathrm{C}), 127.0(\mathrm{CH}), 122.9(\mathrm{CH}), 120.6(\mathrm{C})$, $118.6(\mathrm{C} \times 2), 116.8(\mathrm{CH}), 61.2\left(\mathrm{OCH}_{2}\right), 36.2\left(\mathrm{CH}_{2}\right)$, $28.1\left(\mathrm{CH}_{2}\right), 15.5\left(\mathrm{CH}_{3}\right), 14.2\left(\mathrm{CH}_{3}\right)$. HRMS $\left(\mathrm{ESI}^{+}\right)$: calcd for $\mathrm{C}_{19} \mathrm{H}_{17} \mathrm{NNaO}_{6} \mathrm{~S}_{2}\left(\mathrm{MNa}^{+}\right)$, 442.0395; found, 442.0382. Anal. Calcd. for $\mathrm{C}_{19} \mathrm{H}_{17} \mathrm{NO}_{6} \mathrm{~S}_{2}$ : C, 54.40; H, 4.08; N, 3.34; S, 15.29; found: C, 54.42; H, 4.11; N, 3.37; S, 15.26 .

\section{3f Ethyl 2-(5-\{[6-(tert-butyl)-2-oxo-2H-3-} chromenyl] sulfanyl\}-4-nitro-3-thienyl)acetate 9g:

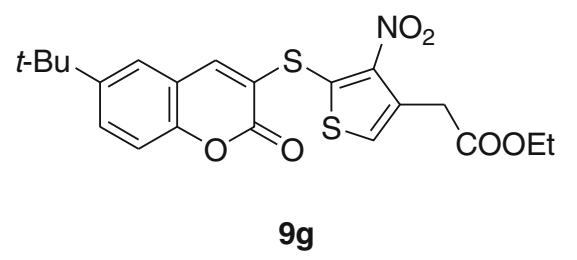

Following the general procedure described above, the reaction of 5-tert-butyl-2-hydroxybenzaldehyde 8 g $(0.057 \mathrm{~g}, 0.32 \mathrm{mmol})$ and ethyl 4-\{[4-(2-ethoxy2-oxoethyl)-3-nitro-2-thienyl]sulfanyl \}-3-oxobutanoate 5 a $(0.1 \mathrm{~g}, 0.27 \mathrm{mmol})$ in the presence of piperidine $(0.001 \mathrm{~g}, 0.1 \mathrm{~mol} \%)$ stirred at $\mathrm{rt}$ for $36 \mathrm{~h}$ furnished $0.076 \mathrm{~g}$ of ethyl 2-(5-\{[6-(tert-butyl)-2-oxo-2H-3chromenyl]sulfanyl\}-4-nitro-3-thienyl)acetate $\mathbf{9 g}$ as yellow colour solid in $64 \%$ yield. Mp: $154-156^{\circ} \mathrm{C}$ (MeOH); UV $\lambda_{\max }(\mathrm{MeOH}): 274 \mathrm{~nm}(\log \varepsilon=4.1)$, $372 \mathrm{~nm}(\log \varepsilon=3.7)$; IR $v_{\max }(\mathrm{KBr}): 1738$ (CO), 1546,
1492, $1319\left(\mathrm{NO}_{2}\right), 1091,883,740 \mathrm{~cm}^{-1} \cdot{ }^{1} \mathrm{H}$ NMR $\delta$ (400 MHz, $\left.\mathrm{CDCl}_{3} ; \mathrm{Me}_{4} \mathrm{Si}\right): 8.21(\mathrm{~s}, 1 \mathrm{H}, \mathrm{CH}), 7.48$ (d, $J=8.1 \mathrm{~Hz}, 1 \mathrm{H}, \mathrm{CH}), 7.32(\mathrm{~d}, J=8.7 \mathrm{~Hz}, 1 \mathrm{H}, \mathrm{CH})$, $7.29(\mathrm{~d}, J=8.6 \mathrm{~Hz}, 1 \mathrm{H}, \mathrm{CH}), 6.98(\mathrm{~s}, 1 \mathrm{H}, \mathrm{CH}), 4.14$ (q, $\left.J=7.2 \mathrm{~Hz}, 2 \mathrm{H}, \mathrm{OCH}_{2}\right), 3.98\left(\mathrm{~s}, 2 \mathrm{H}, \mathrm{CH}_{2}\right), 1.27(\mathrm{~s}$, $\left.9 \mathrm{H}, \mathrm{CH}_{3}\right), 1.23\left(\mathrm{t}, J=7.2 \mathrm{~Hz}, 3 \mathrm{H}, \mathrm{CH}_{3}\right) ;{ }^{13} \mathrm{C} \mathrm{NMR} \delta$ (100 MHz, $\mathrm{CDCl}_{3} ; \mathrm{Me}_{4} \mathrm{Si}$ ): 169.9 (C), 158.1 (C), 153.1 $(\mathrm{CH}), 152.3(\mathrm{C}), 148.2(\mathrm{C}), 140.7(\mathrm{C}), 135.0(\mathrm{CH})$, $134.6(\mathrm{C}), 130.8(\mathrm{C}), 128.8(\mathrm{CH}), 124.6(\mathrm{CH}), 118.5$ (C), $118.4(\mathrm{C}), 116.2(\mathrm{CH}), 60.4\left(\mathrm{OCH}_{2}\right), 35.4\left(\mathrm{CH}_{2}\right)$, $31.6\left(\mathrm{CH}_{3} \times 3\right), 26.3(\mathrm{C}), 14.0\left(\mathrm{CH}_{3}\right)$. HRMS $\left(\mathrm{ESI}^{+}\right)$: calcd for $\mathrm{C}_{21} \mathrm{H}_{21} \mathrm{NNaO}_{6} \mathrm{~S}_{2}\left(\mathrm{MNa}^{+}\right), 470.0708$; found, 470.0699. Anal. Calcd. for $\mathrm{C}_{21} \mathrm{H}_{21} \mathrm{NO}_{6} \mathrm{~S}_{2}: \mathrm{C}, 56.36 ; \mathrm{H}$, $4.73 ; \mathrm{N}, 3.13 ; \mathrm{S}, 14.33$; found: $\mathrm{C}, 56.33 ; \mathrm{H}, 4.72 ; \mathrm{N}$, $3.09 ; \mathrm{S}, 14.31$

2.3g Ethyl 2-\{5-[(7-methoxy-2-oxo-2H-3chromenyl) sulfanyl]-4-nitro-3-thienyl\}acetate $\mathbf{9 h}$ :<smiles>CCOC(=O)Cc1csc(Sc2cc3ccc(OC)cc3oc2=O)c1[N+](=O)[O-]</smiles>

$9 h$

Following the general procedure described above, the reaction of 4-methoxy-2-hydroxybenzaldehyde 8h $(0.049$ g, $0.32 \mathrm{mmol})$ and ethyl 4-\{[4-(2-ethoxy2-oxoethyl)-3-nitro-2-thienyl]sulfanyl $\}$-3-oxobutanoate $5 \mathbf{a}(0.1 \mathrm{~g}, 0.27 \mathrm{mmol})$ in the presence of piperidine $(0.001 \mathrm{~g}, 0.1 \mathrm{~mol} \%)$ stirred at $\mathrm{rt}$ for $34 \mathrm{~h}$ furnished $0.085 \mathrm{~g}$ of ethyl 2-\{5-[(7-methoxy-2-oxo- $2 \mathrm{H}-3-$ chromenyl)sulfanyl]-4-nitro-3-thienyl $\}$ acetate $\mathbf{9 h}$ as yellow colour solid in $73 \%$ yield. Mp: $182-183^{\circ} \mathrm{C}$ $(\mathrm{MeOH}) ; \mathrm{UV} \lambda_{\max }(\mathrm{MeOH}): 276 \mathrm{~nm}(\log \varepsilon=4.3)$, $372 \mathrm{~nm}(\log \varepsilon=3.8)$; IR $v_{\max }(\mathrm{KBr}): 1740(\mathrm{CO}), 1546$, 1492, $1319\left(\mathrm{NO}_{2}\right), 1091,883,740 \mathrm{~cm}^{-1} \cdot{ }^{1} \mathrm{H}$ NMR $\delta$ (400 MHz, $\left.\mathrm{CDCl}_{3} ; \mathrm{Me}_{4} \mathrm{Si}\right): 8.14(\mathrm{~s}, 1 \mathrm{H}, \mathrm{CH}), 7.34$ (d, $J=8.8 \mathrm{~Hz}, 1 \mathrm{H}, \mathrm{CH}), 7.21(\mathrm{~d}, J=6.4 \mathrm{~Hz}, 1 \mathrm{H}$, $\mathrm{CH}), 7.0(\mathrm{~s}, 1 \mathrm{H}, \mathrm{CH}), 6.95(\mathrm{~s}, 1 \mathrm{H}, \mathrm{CH}), 4.18(\mathrm{q}$, $\left.J=7.2 \mathrm{~Hz}, 2 \mathrm{H}, \mathrm{OCH}_{2}\right), 3.89\left(\mathrm{~s}, 2 \mathrm{H}, \mathrm{CH}_{2}\right), 3.87(\mathrm{~s}$, $\left.3 \mathrm{H}, \mathrm{CH}_{3}\right), 1.28\left(\mathrm{t}, J=7.2 \mathrm{~Hz}, 3 \mathrm{H}, \mathrm{CH}_{3}\right) ;{ }^{13} \mathrm{C} \mathrm{NMR} \delta$ (100 MHz, $\mathrm{CDCl}_{3} ; \mathrm{Me}_{4} \mathrm{Si}$ ): 171.7 (C), 169.8 (C), 158.7

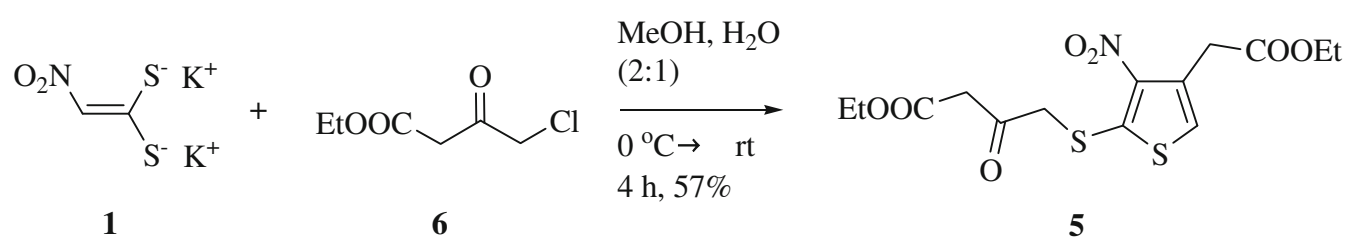

Scheme 2. Reaction of dipotassium salt 1 with ethyl 4-chloroacetoacetate 6. 


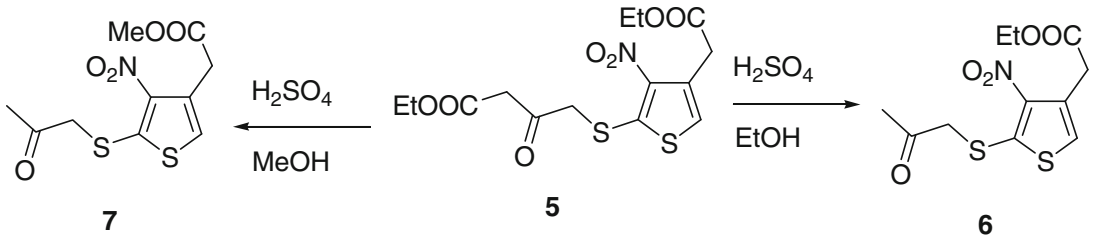

Scheme 3. Solvolysis of 3-nitrothiophene 5.

(C), $156.5(\mathrm{C}), 149.9(\mathrm{CH}), 149.0(\mathrm{C}), 131.2(\mathrm{C}), 123.2$ $(\mathrm{CH}), 121.7(\mathrm{CH}), 120.9(\mathrm{C}), 119.0(\mathrm{C}), 118.1(\mathrm{CH})$, $109.9(\mathrm{CH}), 106.8(\mathrm{C}), 61.3\left(\mathrm{OCH}_{2}\right), 55.9\left(\mathrm{OCH}_{3}\right)$, $36.2\left(\mathrm{CH}_{2}\right), 14.2\left(\mathrm{CH}_{3}\right)$. HRMS $\left(\mathrm{ESI}^{+}\right)$: calcd for $\mathrm{C}_{18} \mathrm{H}_{15} \mathrm{NNaO}_{7} \mathrm{~S}_{2}\left(\mathrm{MNa}^{+}\right)$, 444.0188; found, 444.0179. Anal. Calcd. for $\mathrm{C}_{18} \mathrm{H}_{15} \mathrm{NO}_{7} \mathrm{~S}_{2}: \mathrm{C}, 51.30 ; \mathrm{H}, 3.59 ; \mathrm{N}$, 3.32; S, 15.22; found: C, 50.29; H, 3.62; N, 3.35; S, 15.19 .

2.3h Ethyl 2-\{5-[(6-chloro-7-methyl-2-oxo-2H-3chromenyl)sulfanyl]-4-nitro-3-thienyl\}acetate 9i:<smiles>CCOC(=O)Cc1csc(Sc2cc3cc(Cl)c(C)cc3oc2=O)c1[N+](=O)[O-]</smiles>

$9 \mathbf{i}$

Following the general procedure described above, the reaction of 3-chloro-6-hydroxy-2-methylbenzaldehyde $8 \mathbf{i}(0.024 \mathrm{~g}, 0.16 \mathrm{mmol})$ and ethyl 4-\{[4-(2-ethoxy2-oxoethyl)-3-nitro-2-thienyl]sulfanyl \}-3-oxobutanoate $5 \mathbf{a}(0.05 \mathrm{~g}, 0.13 \mathrm{mmol})$ in the presence of piperidine $(0.001 \mathrm{~g}, 0.1 \mathrm{~mol} \%)$ stirred at $\mathrm{rt}$ for $36 \mathrm{~h}$ furnished $0.026 \mathrm{~g}$ of ethyl 2-\{5-[(6-chloro-7-methyl-2-oxo- $2 \mathrm{H}$ 3-chromenyl)sulfanyl]-4-nitro-3-thienyl $\}$ acetate $\mathbf{9 i}$ as yellow colour solid in $43 \%$ yield. Mp: $189-190^{\circ} \mathrm{C}$ (MeOH); UV $\lambda_{\max }(\mathrm{MeOH}): 275 \mathrm{~nm}(\log \varepsilon=4.3)$, $382 \mathrm{~nm}(\log \varepsilon=3.8)$; IR $v_{\max }(\mathrm{KBr}): 1740(\mathrm{CO}), 1546$, 1492, $1319\left(\mathrm{NO}_{2}\right), 1091,883,740 \mathrm{~cm}^{-1} .{ }^{1} \mathrm{H}$ NMR $\delta$ (400 MHz, $\left.\mathrm{CDCl}_{3} ; \mathrm{Me}_{4} \mathrm{Si}\right): 8.4(\mathrm{~s}, 1 \mathrm{H}, \mathrm{CH}), 7.59$ (d, $J=9.2 \mathrm{~Hz}, 1 \mathrm{H}, \mathrm{CH}), 7.21(\mathrm{~d}, J=8.8 \mathrm{~Hz}, 1 \mathrm{H}, \mathrm{CH}), 7.0$ (s, 1H, CH), 4.18 (q, J = 7.2 Hz, 2H, OCH $)_{2}, 3.89$ (s, $\left.2 \mathrm{H}, \mathrm{CH}_{2}\right), 2.59\left(\mathrm{~s}, 3 \mathrm{H}, \mathrm{CH}_{3}\right), 1.28(\mathrm{t}, J=7.2 \mathrm{~Hz}, 3 \mathrm{H}$, $\left.\mathrm{CH}_{3}\right) ;{ }^{13} \mathrm{C}$ NMR $\delta\left(100 \mathrm{MHz}, \mathrm{CDCl}_{3} ; \mathrm{Me}_{4} \mathrm{Si}\right): 169.7$
(C), $157.6(\mathrm{C}), 153.5(\mathrm{C}), 146.6(\mathrm{CH}), 144.5(\mathrm{C}), 134.4$ (C), $133.9(\mathrm{CH}), 131.3(\mathrm{C}), 130.9(\mathrm{C}), 130.5(\mathrm{C}), 123.4$ (CH), $121.6(\mathrm{C}), 118.8(\mathrm{C}), 115.9(\mathrm{CH}), 61.3\left(\mathrm{OCH}_{2}\right)$, $36.1\left(\mathrm{CH}_{2}\right), 15.6\left(\mathrm{CH}_{3}\right), 14.2\left(\mathrm{CH}_{3}\right)$. HRMS $\left(\mathrm{ESI}^{+}\right)$: calcd for $\mathrm{C}_{18} \mathrm{H}_{14} \mathrm{ClNNaO}_{6} \mathrm{~S}_{2}\left(\mathrm{MNa}^{+}\right)$, 461.9849; found, 461.9832. Anal. Calcd. for $\mathrm{C}_{18} \mathrm{H}_{14} \mathrm{ClNO}_{6} \mathrm{~S}_{2}$ : C, 49.15; $\mathrm{H}, 3.21 ; \mathrm{Cl}, 8.06 ; \mathrm{N}, 3.18 ; \mathrm{O}, 21.82 ; \mathrm{S}, 14.58$; found: C, 49.11; H, 3.20; Cl, 8.07; N, 3.19; O, 21.78; S, 14.56.

2.3i Ethyl 2-\{4-nitro-5-[(3-oxo-3H-benzo[f]chromen2-yl)sulfanyl]-3-thienyl\}acetate 11:<smiles>CCOC(=O)Cc1csc(Sc2cc3c(ccc4ccccc43)oc2=O)c1[N+](=O)[O-]</smiles>

11

Following the general procedure described above, the reaction of 2- hydroxy-1-napthaldehyde $\mathbf{1 0}$ (0.055 g, $0.32 \mathrm{mmol}$ ) and ethyl 4-\{[4-(2-ethoxy-2-oxoethyl)3-nitro-2-thienyl]sulfanyl\}-3-oxobutanoate 5a (0.1 g, $0.27 \mathrm{mmol})$ in the presence of piperidine $(0.001 \mathrm{~g}$, $0.1 \mathrm{~mol} \%$ ) stirred at $\mathrm{rt}$ for $36 \mathrm{~h}$ furnished $0.067 \mathrm{~g}$ of ethyl 2-\{4-nitro-5-[(3-oxo-3 H-benzo[ $f]$ chromen-2yl)sulfanyl]-3-thienyl $\}$ acetate $\mathbf{1 1}$ as yellow colour solid in $57 \%$ yield. $\mathrm{Mp}: 196-198^{\circ} \mathrm{C}(\mathrm{MeOH})$; $\mathrm{UV} \lambda_{\max }$ (MeOH): $276 \mathrm{~nm}(\log \varepsilon=4.3), 394 \mathrm{~nm}(\log \varepsilon=$ 3.8); IR $v_{\max }(\mathrm{KBr}): 1740(\mathrm{CO}), 1546,1492,1319$ $\left(\mathrm{NO}_{2}\right), 1091,883,740 \mathrm{~cm}^{-1} .{ }^{1} \mathrm{H}$ NMR $\delta(400 \mathrm{MHz}$, $\left.\mathrm{CDCl}_{3} ; \mathrm{Me}_{4} \mathrm{Si}\right): 9.0(\mathrm{~s}, 1 \mathrm{H}, \mathrm{CH}), 8.26(\mathrm{~d}, J=$ $8.0 \mathrm{~Hz}, 1 \mathrm{H}, \mathrm{CH}), 8.11(\mathrm{~d}, J=12.0 \mathrm{~Hz}, 1 \mathrm{H}$, $\mathrm{CH}), 8.0(\mathrm{~d}, J=8.0 \mathrm{~Hz}, 1 \mathrm{H}, \mathrm{CH}), 7.76(\mathrm{t}$, $J=8.0 \mathrm{~Hz}, 1 \mathrm{H}, \mathrm{CH}), 7.64(\mathrm{t}, J=8.0 \mathrm{~Hz}, 1 \mathrm{H}, \mathrm{CH})$,<smiles>CCOC(=O)CC(=O)CSc1scc(CC(=O)OCC)c1[N+](=O)[O-]</smiles>

5

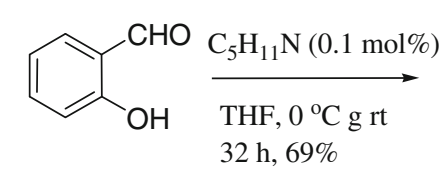

$8 \mathbf{a}$

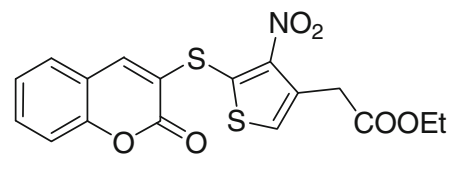

9a

Scheme 4. Reaction of 2-hydroxybenzaldehyde 8a with $\beta$-keto ester unit in parent thiophene 5a. 
Table 1. Transformation of $\beta$-keto ester 5a to coumarins $9 \mathbf{a}-\mathbf{i}$.

Entry 2-Hydroxy benzaldehy

1

$8 \mathbf{a}$<smiles>O=Cc1cc(Cl)ccc1O</smiles>

2

3

4

5

6<smiles>O=Cc1cc(Br)ccc1O</smiles>

8c<smiles>COc1ccc(O)c(C=O)c1</smiles>

8d<smiles>Cc1ccc(O)c(C=O)c1</smiles>

$8 e$<smiles>CCc1ccc(O)c(C=O)c1</smiles>

$8 f$<smiles>CC(C)(C)c1ccc(O)c(C=O)c1</smiles>

7<smiles>COc1ccc(C=O)c(O)c1</smiles>

8

$8 h$<smiles>Cc1c(Cl)ccc(O)c1C=O</smiles>

4-Nitro-3-thienyl-2 $H$-chromenes 9a-i

Time (h)

Yield (\%)<smiles>CCOOC(=O)Cc1csc(Sc2cc3ccccc3oc2=O)c1[N+](=O)[O-]</smiles>

9a<smiles>CCOOC(=O)Cc1csc(Sc2cc3cc(Cl)ccc3oc2=O)c1[N+](=O)[O-]</smiles>

$9 b$

34

66<smiles>CCOOC(=O)Cc1csc(Sc2cc3cc(Br)ccc3oc2=O)c1[N+](=O)[O-]</smiles>

9c<smiles>CCOC(=O)Cc1csc(Sc2cc3cc(OC)ccc3oc2=O)c1[N+](=O)[O-]</smiles>

9d<smiles>CCOOC(=O)Cc1csc(Sc2cc3cc(C)ccc3oc2=O)c1[N+](=O)[O-]</smiles>

9 e<smiles>CCOC(=O)Cc1csc(Sc2cc3cc(CC)ccc3oc2=O)c1[N+](=O)[O-]</smiles>

9f<smiles>CCOC(=O)Cc1csc(Sc2cc3cc(C(C)(C)C)ccc3oc2=O)c1[N+](=O)[O-]</smiles>

36

64<smiles>CCOOC(=O)Cc1csc(Sc2cc3ccc(OC)cc3oc2=O)c1[N+](=O)[O-]</smiles>

9h<smiles>CCOOC(=O)Cc1csc(Sc2cc3c(C)c(Cl)ccc3oc2=O)c1[N+](=O)[O-]</smiles> 


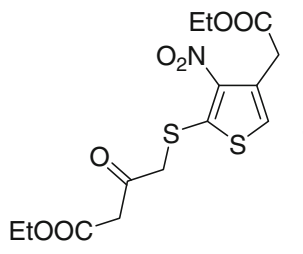

5

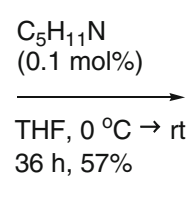

10

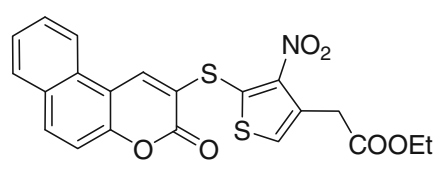

11

Scheme 5. Reaction of 2-hydroxy-1-napthaldehyde $\mathbf{1 0}$ with $\beta$-keto ester unit in parent thiophene $\mathbf{5 a}$.

$7.52(\mathrm{t}, J=8.0 \mathrm{~Hz}, 1 \mathrm{H}, \mathrm{CH}), 6.99(\mathrm{~s}, 1 \mathrm{H}, \mathrm{CH}), 4.19$ $\left(\mathrm{q}, J=8.0 \mathrm{~Hz}, 2 \mathrm{H}, \mathrm{OCH}_{2}\right), 3.90\left(\mathrm{~s}, 2 \mathrm{H}, \mathrm{CH}_{2}\right), 1.28$ $\left(\mathrm{t}, J=8.0 \mathrm{~Hz}, 3 \mathrm{H}, \mathrm{CH}_{3}\right) ;{ }^{13} \mathrm{C} \mathrm{NMR} \delta(100 \mathrm{MHz}$, $\mathrm{CDCl}_{3} ; \mathrm{Me}_{4} \mathrm{Si}$ ): 169.9 (C), 158.6 (C), 155.2 (C), 147.1 $(\mathrm{CH}), 146.8(\mathrm{C}), 135.5(\mathrm{CH}), 131.3(\mathrm{C} \times 2), 130.4(\mathrm{C})$, $129.3(\mathrm{CH}), 129.2(\mathrm{CH}), 128.8(\mathrm{C}), 126.8(\mathrm{CH}), 122.8$ $(\mathrm{CH}), 121.4(\mathrm{CH}), 119.1(\mathrm{C}), 116.8(\mathrm{CH}), 113.2(\mathrm{C})$, $61.3\left(\mathrm{OCH}_{2}\right), 36.3\left(\mathrm{CH}_{2}\right), 14.2\left(\mathrm{CH}_{3}\right)$. HRMS $\left(\mathrm{ESI}^{+}\right)$: calcd for $\mathrm{C}_{21} \mathrm{H}_{15} \mathrm{NNaO}_{6} \mathrm{~S}_{2}\left(\mathrm{MNa}^{+}\right), 464.0238$; found, 464.0224. Anal. Calcd. for $\mathrm{C}_{21} \mathrm{H}_{15} \mathrm{NO}_{6} \mathrm{~S}_{2}$ : C, 57.13; H, 3.42 ; N, 3.17; S, 14.53; found: C, 57.11; H, 3.39; N, 3.14; S, 14.49 .

\section{Results and discussion}

The reaction of ethyl chloroacetoacetate $\mathbf{6}$ with dipotassium nitroketenedithioacetate 1 provided 3nitrothiopene 5a as the only product in 57\% yield (scheme 2). The structure of the thiophene 5a was established on the basis of spectroscopic (IR, ${ }^{1} \mathrm{H}$ NMR, ${ }^{13} \mathrm{C}$ NMR, 2D NMR and HRMS), analytical data and single crystal X-ray structure determination. ${ }^{9}$ As anticipated from the assigned structure, ${ }^{1} \mathrm{H}$ NMR spectrum of $\mathbf{5 a}$ displayed four singlets for three methylenes $(\delta$ $3.66,3.85,4.11 \mathrm{ppm})$ and one for $\mathrm{C} 5 \mathrm{H}(\delta 7.01 \mathrm{ppm})$. The ${ }^{1} \mathrm{H}$ NMR spectrum also revealed occurrence of the keto-enol tautomerism to the extent of 85:15 where keto-form predominated.

The thiophene 5a, prepared in this study possesses multiple functional groups like ketone, ester and nitrogroups. Particularly, there are three active methylenes flanked by three carbonyl carbons. Initially, we treated thiophene 5a with EtOH in presence of a catalytic amount of $\mathrm{H}_{2} \mathrm{SO}_{4}$. The product was the anticipated methyl ketone 7 (scheme 3). Reaction of with $\mathrm{MeOH}$ in presence of catalytic amount of $\mathrm{H}_{2} \mathrm{SO}_{4}$ provided 7a, the decarboethoxylated and trans-esterification product (scheme 3 ).

Next, we considered transformation of $\mathbf{5 a}$ into coumarin and 3-nitrothiophene conjugates by reaction of the $\beta$-keto ester moiety in 5a with 2- hydroxybenzaldehyde. Many natural products with coumarin (benzopyrone) motif have been isolated from plant sources and such molecules show immense biological activity. ${ }^{10}$ In addition, coumarin and its C3-substitution products found clinical medical applications as blood thinners or as anticoagulants. ${ }^{11}$ Some coumarin derivatives are triplet sensitizers and are in use as dye lasers. ${ }^{12}$ Classical routes to coumarins include Pechmann, ${ }^{13,14}$ Knoevenagel, ${ }^{15}$ Perkin, ${ }^{16}$ Reformatsky ${ }^{17}$ and Wittig ${ }^{18}$ condensation reactions. Thus, thiophene 5a was subjected to Pechmann condensation with 2-hydroxybenzaldehyde (salicylaldehyde) 8a to produce a C3-nitrothiophene substituted coumarin 9a. The reaction conducted in presence of piperidine in THF produced the coumarin, ethyl 2-\{4-nitro-5-[(2oxo-2 $\mathrm{H}$-3-chromenyl)sulfanyl]-3-thienylacetate $\mathbf{9 a}$ as the only product in $69 \%$ yield (scheme 4 ). By changing solvents and bases systematically, we found that the transformation works well in presence of a catalytic amount of piperidine $(0.1 \mathrm{~mol} \%)$ in THF at rt. Even though catalytic amount of piperidine demanded longer reaction time (table 1), the reaction was cleaner and product isolation was facile. The coumarin 9a was characterized on the basis of spectroscopic (IR, ${ }^{1} \mathrm{H}$ NMR, ${ }^{13} \mathrm{C}$ NMR, 2D NMR and HRMS) and analytical data. Three singlets at $\delta 3.89 \mathrm{ppm}$ for two hydrogens, at $\delta 7.02$ for one hydrogen and at $8.22 \mathrm{ppm}$ for one hydrogen assignable to methylene, thiophene $\mathrm{C} 5 \mathrm{H}$ and coumarin $\mathrm{C} 4 \mathrm{H}$ respectively, were notable signals in the ${ }^{1} \mathrm{H}$ NMR spectrum.

The Pechmann transformation of thiophene 5a into coumarin 9a proved to be quite general for nine 2hydroxybenzaldehydes $\mathbf{8} \mathbf{a}-\mathbf{i}$ and the coumarin products 9a-i were obtained in $42-73 \%$ yield (table 1).

Scope of the present 3-nitrothiophene substituted coumarin synthesis could be further extended by making 2-hydroxy-1-naphthaldehyde $\mathbf{1 0}$ participate in the condensation reaction (scheme 5). This reaction provided coumarin 11 in 57\% yield. Spectroscopic data supported structure. Particularly noteworthy is the singlet for $\mathrm{C} 4 \mathrm{H}$ of the coumarin ring in the ${ }^{1} \mathrm{H}$ NMR spectrum which appeared at $\delta 9.1 \mathrm{ppm}$. 
Mechanistically, the transformation is interesting as one acetate unit in 5a was lost during coumarin formation. ${ }^{19}$ Moreover, the active methylene adjacent to $\mathrm{C} 2 \mathrm{~S}$, instead of that of the $\beta$-keto ester moiety was utilized in the coumarin synthesis. This is in contrast to general expectation that $\beta$-keto ester moiety in 5a would react in preference over the methylene flanked by $\mathrm{C} 2 \mathrm{~S}$ and $\mathrm{CO}$ groups during coumarin formation. Perhaps, under the reaction conditions, the carbanion generated on $\mathrm{C} 2 \mathrm{~S}$ methylene is more reactive than the carbanion generated on the $\beta$-keto ester moiety.

\section{Conclusions}

Our studies clearly delineated the alkylation of the salt $\mathbf{1}$ with ethyl 4-chloroacetoacetate $\mathbf{6}$ to provide the thiophene 5a which could be utilized for the synthesis of novel coumarins $\mathbf{9}$ and $\mathbf{1 1}$ with 3-nitrothiophene moiety at $\mathrm{C} 3$ position. Overall, we have demonstrated a facile two-step synthesis of hybrid heterocyles incorporating biologically important 3-nitrothiophene and coumarin units starting from inexpensive and readily available chemicals and reagents. Furthermore, we have demonstrated that the one of the two esters in $\mathbf{5 a}$ can be selectively decarboethoxylated to provide methyl ketones as well trans-esterification products.

\section{Acknowledgements}

HSPR thanks University Grants Commission for UGCSAP, Department of Science and Technology for Fund for Improvement of S\&T Infrastructure in Universities and Higher Educational Institutions (FIST) and Council of Scientific and Industrial Research (CSIR) for financial support. VK thanks CSIR for research fellowship. We thank Prof. A Srikrishna, Indian Institute of Science (IISc), Bangalore, for providing spectral data.

\section{References}

1. (a) Metzner P and Thuillier A 1994 Sulfur reagents in organic chemistry. (London: Academic Press) (b) Kolb M 1990 Synthesis 171 (c) Tominaga Y and Matsuda Y 1985 J. Heterocycl. Chem. 22937 (d) Misra N C, Panda K, Ila H and Junjappa H 2007 J. Org. Chem. 721246 (e) Rao H S P and Sivakumar S 2005 J. Org. Chem. 704524 (f) Terang N, Mehta B, Ila H and Junjappa H 1998 Tetrahedron 5412973 (g) Hsu A C-T, Peter O-G, Joseph R W and Clifford L B 1997 Eur. Pat. Appl. EP 765870, Chem. Abstr. 1997126263848 (h) Gompper R and Schaefer H 1967 Chem. Ber. 100591 (i) Freund E 1919 Chem. Ber. 52B 542
2. Rao H S P, Sakthikumar L and Shreedevi S 2002 Sulfur Lett. 25207

3. Rao H S P, Sakthikumar L, Vanitha, S and Sivakumar, S 2003 Tetrahedron Lett. 444701

4. Rao H S P 2008 Indian J. Chem., Sect B. 47B 272

5. Rao H S P and Vasantham K 2009 J. Org. Chem. 74 6847

6. Batchu C 2008 J. Sulfur Chem. 29 187-240 (b) Russell R K 1996 Comprehensive Heterocyclic Chemistry II (ed.) (New York: Pergamon Press) 2 pp. 679-729 (c) Rajappa S and Natekar M V 1996 Comprehensive Heterocyclic Chemistry II A R Katritzky, C W Rees, E F V Scriven (eds) (New York: Pergamon Press) 2 pp 491 (d) Blicke F F 1952 Biological and Pharmacological Activity of Thiophene and its Derivatives H D Hartough (ed.) (New York: Interscience) p. 29

7. (a) Morley J O and Matthews T P 2006 Org. Biomol. Chem. 4359 (b) Campaigne E 1994 Comprehensive Heterocyclic Chemistry 4922

8. (a) Wynberg H 1960 Chem. Rev. 60164 (b) Wynberg H and Meijer E 1982 Org. Reactions 28 136 (c) Wynberg H 1991 The Reimer-Tiemann reaction in comp. Org. Synth. (eds). Trost, B. M.; Fleming, I (Oxford: Pergamon) 2269

9. Details of X-ray single crystal structure determination of 5 was deposited at CCDC (deposition No. 633059)

10. (a) Hepworth J D Gabbutt C D Heron B M in 1996 Comprehensive heterocyclic chemistry-II A R Katritzky, C W Rees and E F V Scriven (eds). 5417 (b) Hepworth J D in 1984 Comprehensive heterocyclic chemistry A R Katritzky, C W Rees (eds). 3799 (c) Murray R D H Mendez Jand Brown S A 1982 The natural coumarins: Occurrence, chemistry and biochemistry (New York: John Wiley and Sons) 227

11. O'Kennedy R and Thornes R D 1997 Coumarins: Biology, applications and mode of action (Chichester UK: Wiley)

12. (a) Fluorescent indicators: Brun M P Bischoff $\mathrm{L}$ and Garbay C 2004 Angew Chem. Int. Ed. 433432 (b) Laser technology: Sekar N 2003 Colourage 5055 (c) Fluorescent chemo-sensors: Chen C-T and Huang W-P $2002 \mathrm{~J}$. Am. Chem. Soc. 1246246

13. (a) von Pechmann and H Duisberg C 1883 Ber. Dtsch. Chem. Ges. 162119 (b) von Pechmann Hand Duisberg C 1884 Chem. Ber. 17929 (c) Russel A and Fyre J R 1941 Org. Synth. 2122 (d) Sethna S and Phadke R 1953 Org. React. 71 (e) Rabjohn N 1976 Org. React. 24261 (e) Sugino T and Tanaka K 2001 Chemistry Lett. 513

14. Rao H S P and Sivakumar S 2006 J. Org. Chem. 718715

15. (a) Jones G 1967 Org. React. 15204 (b) Brufola G, Fringuelli F, Piermatti O and Pizzo F 1996 Heterocycles 431257

16. Johnson J R 1942 Org. React. 1210

17. Shriner R L 1942 Org. React. 11

18. (a) Narasimhan N S, Mali R S and Barve M V 1979 Synthesis 906 (b) Harayama T, Nakatsuka K, Katsuro K, Nishioka H, Murakami K and Fuji M 1993 Chem. Express 8245 (c) Yavar I, Hekmat-Shoar R and Zonouzi A 1998 Tetrahedron Lett. 392391

19. Yamamoto T, Yamashita A and Numoto N 1992 U.S.patent 6 pp. CAN 117:25937 AN 1992:425937 